\title{
Biblische und klassische Urgeschichte.
}

\author{
Von Prof. Dr. Armin Ehrenzweig in Graz.
}

\section{Die Verbreitung der Urgeschichte.}

In einer Abbandlung über Kain und Lamech $^{\mathbf{x}}$ habe ich gezeigt, daß die Geschichte von Romulus und Remus nur eine andere Fassung der biblischen Brudermordsage ist. Der aufgefundene Faden soll nun weiterverfolgt, insbesondere das Ende des Romulus von dem neuen Standpunkte aus beleuchtet werden. Zunächst aber drängt sich der Wunsch auf, irgendeine Vorstellung von'dem Wege zu gewinnen, der von der kanaanäischen zur römischen Sage hinüberführt. Denn wer eine vergleichende Darstellung der Sagen so weit auseinanderliegender Völker unternimmt, und nicht minder, wer die unternommene beurteilt, steht immer im Banne irgendeiner Vorstellung von-der Art, wie ein solcher Zusammenhang überhaupt zu denken wäre.

Die unsicheren Vermutungen, die ich hierüber in meiner ersten Abhandlung gewagt habe, können genauerer Prüfung nicht standhalten. Sie erklären eine Übereinstimmung nicht, die sich auf die ganze Ordnung einer weitläufigen Erzählung und zugleich auch auf einzelne nebensächliche Züge, ja auf die Art der D̉arstellung erstreckt. Hier muß literarische, gelehrte Übertragung im Spiele sein.

Wie in so vielen andern wirklichen oder vermeintlichen Wissenschaften sind die Babylonier auch in der Geschichte die Lehrmeister anderer Völker gewesen. Ihre Berichte über die Sintflut und über die vorausgegangene Urgeschichte des Menschengeschlechtes fanden nicht weniger Glauben als ihre astronomischen Beobachtungen oder ihre astrologische Scheinweisheit. Rühmten sich doch die Babylonier des Besitzes vorsintflutlicher Schriften ${ }^{2}$. Kein Verdacht wagte sich an diese ehrwürdigen. Geschichtsquellen heran. Deshalb wanderte die babylonische Sintfluterzählung und mit ihr die ganze Geschichte des vorsintflutlichen Zeitalters von Volk zu Volk, Auch der Jahwist oder

In dieser Zeitschrift 35, 1 .

2 Zmamern, Die Keilinschriften und das Alte Testament ${ }^{3} \mathrm{~S} .537$.

Zeitschr. f. d. alttest. Wiss. Jahrg. 38. $1989 / 20$. 
wie man sonst den Schriftsteller nennen mag, der zuerst die Geschichte des Volkes Israel schrieb, begann seine Darstellung nach der Sitte jener Zeit mit der Erschaffung der Welt. Er benützte die damals bereits in ganz Vorderasien verbreitete Darstellung der Urgeschichte, nicht weil es ihm bequem war oder weil der Inhalt seinen Absichten entsprach das Gegenteil war der Fall -, sondern weil er als Geschichtschreiber sich an die maßgebenden Quellen halten mußte. Er beanspruchte Glauben und er glaubte selbst an das Erzählte. DaB nach und nach tendenziöse Änderungen seine „wissenschaftliche“ Arbeit entstellt haben, liegt klar zutage, aber der Zusammenhang mit den babylonischen Quellen ist immer noch erkennbar.

In Kleinasien war die Urgeschichte wohlbekannt. Sie wurde hier an verschiedenen Orten, insbesondere in Lykaonien und in dem benachbarten Phrygien lokalisiert. In Griechenland taucht sie bei den genealogischen Schriftstellern etwa im siebenten Jahrhundert auf, in Rom ist́ sie seit dem vierten Jahrhunderte nachweisbar ${ }^{I}$. Die Verbindung zwischen Ost und West - zwischen Kleinasien und Rom - hat wahrscheinlich Delphi hergestellt: Denn diese hochberühmte Orakelstätte pflegte seit alter Zeit nach beiden Richtungen hin lebhaften Verkehr. Die Beziehungen zu Rom reichen der Sage nach bis in die Königszeit hinauf ${ }^{2}$. Nun wissen wir, daß die Delphischen Chronisten von der Sintflut und von den vorsintflutlichen Königen erzählt und daß sie diese Erzählung in ihrer Heimat lokalisiert haben. Als vorsintflutliche Stadt galt ihnen Lykoreia auf dem Parnassos, Delphis angebliche Mutterstadt. Das war die alte Königsstadt des Sintfluthelden Deukalion ${ }^{3}$. Von ihrer Gründung erfahren wir leider recht wenig. Der Gründer war Parnassos, Poseidons und der Kleodora Sohn. Eben dieser Parnassos hat die Weissagung aus dem Vogelfluge erfunden ${ }^{4}$. Diese Nachricht genügt immerhin, die Vermutung zu rechtfertigen, daB in der delphischen Sage von der Gründung der vorsintflutlichen Stadt die Beobachtung des Vogelfuges eine Rolle gespielt habe. Das wäre dann ein Zug, den die römische Gründungssage mit der delphischen gemein hat und den wir um so eher auf Rechnung des griechischen Vorbildes setzen können,

I J. MEsk, Die romische Gründungssiage und Nàvius, Wiener Studien 36, 6.

${ }^{2}$ Als geschichtlich gilt die Sendúng eines Weihgeschenkes von Rom nach Delph im Jahre 396 v; Chr. De SANCtis, Storia dei Romani II 146.

${ }^{3}$ Usener, Sintllutsagen, S. 76 f., JaCoBy, Marmor Parium, S. 3 I.

4 Pausanias X 6. 
als die Art, wie die Auspizien in der römischen Gründungssage behandelt werden, dem 'römischen Ausspizienritus durchaus widerspricht' ${ }^{I}$. Wir besitzen aber auch ein ausdrückliches Zeugnis, das uns von Rom nach Lykoreia zurückweist. In der römischen Sage ist, nämlich von einem Asyle die Rede, das Romulus nach griechischem Muster ${ }^{2}$ eingerichtet haben soll. Den Gott, dem dieses Asyl heilig war, nennt der Annalist Piso $^{3}$ Lucoris. Das ist Lykoreios, der Zeus von Lykoreia. Darüber besteht kein Zweifel ${ }^{4}$.

Leider ist uns von der Geschichte des vorsintflutlichen Lykoreia außer der dürftigen Nachricht von der Auspizienerfindung ihres Gründers nichts erhalten. Es gibt jedoch eine zweite griechische Sage, die sich gleichfalls mit der Gründung der ersten Stadt befaßt: die Sage von der Gründung der arkadischen Stadt Lykosura. Ihre Verwandtschaft mit der delphischen Erzählung ist längst bemerkt worden ${ }^{5}$. Hier aber wird uns eine zusammenhängende Urgeschichte ${ }^{6}$ überliefert, die mit dem ersten Menschen (Pelasgos) beginnt ${ }^{7}$ und bis zur geschichtlichen Zeit hinabreicht. Der zweite arkadische Urvater Lykaon hat Lykosura gegründet. Das war die erste Stadt unter dem Monde, nach, deren Vorbilde dann die Menschen andere Städte $z u$ gründen gelernt haben ${ }^{8}$. Der Name des Gründers, Lykaon, ist nicht griechisch. Bei Homer heißt ein lykischer Fürst so. Im Namen der kleinasiatischen Landschaft Lykaonien erkennen wir leicht den Namen des Stadtgründers wieder. Der Name hängt also nicht mit den griechischen.Wörtern für Wolf oder Licht zusammen, er bezeichnet vielmehr den Eponymus des lykischen Volkes. Der Wolf ist erst bei den Griechen in die Stadtgründungsgeschichte hineingeraten und hat sich da auch bei den Römern behauptet.

Wenden wir uns nun, indem wir den Weg der Urgeschichte nach rückwärts verfolgen, nach Lykaonien, so finden wir sie hier - und zwar in Ikonion - deutlich genug lokalisiert ${ }^{9}$. In Ikonion wurden auf Befehl des Zeus die ersten Menschen aus Lehm gebildet und vom

I Das hat Monmsex, Hist. Schr. I II gezeigt.

2 Servius zu Aen. II 761, VIII 342.

${ }^{3}$ Bei Servius zu Aen. II 761 .

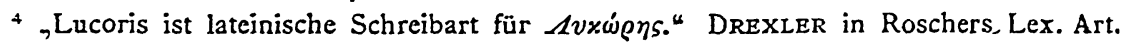
Lykoreus. $\quad$ IMMERWAHR, Kulte und Mythen Arkadiens, S. 22.

- Pausakias Vill i ff.

"Über ihn unten $V$ bei S. 79, Anm. 4. Uber die an Gen 5 erinnernde L.anglebigkeit der arkadischen Ükönige unten VI S. 84, Anm. 5.

- Pausanias vill 38.

9 STEPH. Byz. 'Ixóvıov. 
Winde belebt, hier lebte Nannakos, der phrygische MethuSelah', hier auch jener Flutheld, den die. Griechen Deukalion oder Ogyges ${ }^{2}$ nennen.

Wir befinden uns auf asiatischem Boden, der Zusammenhang der lykisch-phrygischen mit der babylonisch-biblischen Sage bedarf nicht erst des Nachweises, obwohl über den Weg, den die Urgeschichte hier - innerhalb Vorderasiens - zurückgelegt hat, kaum eine Vermutung möglich ist ${ }^{3}$. Nach Rom ist sie wahrscheinlich noch in ihrer echten Gestalt, als Geschichte der Urväter, gelangt. Die römischen Schriftsteller haben ja sogar einen besonderen Ausdruck zur Bezeichnung der Urväter geschaffen: Aborigines. Ein Volk dieses Namens hat es nie gegeben, und die den Alten geläufige Ableitung $n^{a b}$ origine" 4 ist durchaus annehmbar, wenn wir in dem Ausdrucke eben nur eine gelehrte Konstruktion $^{5}$ und nicht eine volkstümliche Bildung erblicken. Die Heimat der Aboriginer suchten die Römer erst, ihrer griechischen Quelle folgend, in Griechenland, dann aber in Italien selbst, und zwar - nach dem Muster von Lykoreia oder Lykosura - im Hochgebirge ${ }^{\circ}$.

Als dann Rom groß genug geworden war, um das Bedürfnis zu empfinden, auch seine geschichtslosen Anfänge nachträglich in das Licht der Geschichte zu rücken, da stellten seine Chroninisten aus mancherlei Quellen die uns so wohl bekannte Königsgeschichte zusammen und sie verwerteten dabei auch die Erzählung von den Urvätern vielleicht gerade deshalb, weil sie dem Volke fremd geblieben war. Vor allem entnahmen sie der Urgeschichte die Erzählung von der Stadtgründung, die nun, aus ihrem alten Zusammenhange gelöst, nicht mehr irgendeine vorsintflutliche Stadt in Griechenland oder in den Abruzzen, sondern die Stadt Rom selbst behandelt. Der Stadtgründer ist damit aus der Reihe der Urväter in ein jüngeres Geschlecht hinabgerückt.

I Uber ihn unten VI.

2 Ogygier ist - nach Stephan yoN Byzanz - ein anderer Name der Lykier.

3 Es ist nicht ausgeschlossen, daß die babylonische, Sage über Kleinasien nach Palastina gewandert ist, oder daB der Jahwist neben einer babylonischen (oder syrischen) Quelle eine kleinasiatische (hethitische) benützt hat. Darauf deutet der Tibarener Kain (Tubalkain) in Gen 4 32. Die Tibarener sind ja ein kleinasiatisches Volk. .

- 4 Eine Anspielung darauf bei Vergil, Aneis 7, 180. Im Sinne von "Stammvater* (Schwegler, Römische Geschichte, S. 599) braucht den Ausdruck Aborigines z. B. Plinius, hist. nat. 4, 36: Tyrii, aborigines Erythriorum.

s O. Keller, Lat. Volksetymologien, S. $20 \mathrm{f}, 35 \mathrm{I}$.

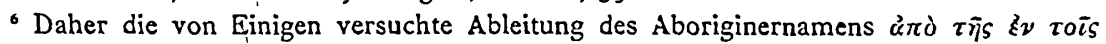

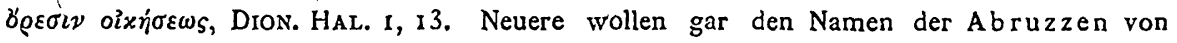
den Aborigines ableiten. Vgl. dagegen DE SANCTIS, Storia I 175. 
Aber dèr älteste Schriftsteller, der ihn kennt - Kallias, eìn Zeitgenosse des Agathokles († 289) - bringt ihn immer noch mit den Aboriginern in Verbindung, ihm ist Romulus ein Sohn des Latinus, "des Königs der Aboriginer" $\mathrm{r}$.

\section{Die Ermordung des Stadtgründers.}

Des Romulus Ende wird uns auf zweierlei Art, beidemal mit vielen Einzelheiten, erzählt. Danach ist Romulus entweder erschlagen oder zu den Göttern entrückt worden. Ist Kain das Urbild des Romulus, so muß die Genesis die Frage lösen, welche von beiden Erzählungen die ursprüngliche sei. Leider schweigt sie über das Ende Kains. Aber daß er ermordet worden ist, läßt sich erraten. Denn die Sage pflegt uns nicht göttliche Offenbarungen zu übermitteln, die keine praktische Bedeutung erlangt haben. Jahwe hatte verkündet: „Wer Kain totschlägt, das wird siebenfach gerochen." Also ist Kain unfehlbar totgeschlagen und sein Tod siebenfach gerächt worden. Das siebenfache Menschenopfer, das in Gibeon zur Erntezeit dargebracht wurde (II Sam 2I), soll an die Ermordung des Urvaters érinnern 2. Die Kainsage beginnt also als Kultlegende des Städtebauopfers und schließt als Kultlegende eines vorisraelitischen Osterfestes. Aber diese beiden Legenden gehören ursprünglich nicht zusammen. Die erste stammt, wie die ganze Urgeschichte, von einem fremden Volke, sie ist in Kanaan nicht lokalisiert (sondern irgendwo im fabelhaften Lande Nod östlich von Eden) - die andere dagegen ist eine einheimische, kanaanäische Festlegende. Jene ist nur den Schriftgelehrten bekannt, diese volkstümlich, im frommen Liede gefeiert. Die Gestalt des ermordeten Urvaters ist also mit der eines kanaanäischen Helden zusammengeflossen.

Des Urvaters Geschichte war ursprünglich gewiß eine einheitliche Legende, eben die Legende des Bauopfers. Aber die Stadtgrụ̈ndung fordert ein doppeltes Bauopfer ${ }^{3}$ : eines bei der Grundsteinlegung, das andere bei oder nach der Vollendung des Baus. Gerne macht die Bausage die beiden Geopferten zu Geschwistern. Das alttestamentliche Geschichtsbuch selbst hat uns eine Erzählung dieser Art bewahrt: Als Hiel die Stadt Jericho baute, da legte er um seinen erstgeborenen Sohn

I Dion. Hal. I 72 ; Momsisen, Hist. Schr. I 3.

2 ZAW 35, 10.

3 Vgl. über das doppelte Bauopfer SARTori, Z. f. Ethnol. 3o, 13. 
Abiram ihren Grund und um seinen jüngsten Sohn Segub setzte er die Türen ${ }^{x}$. In unserem Falle muß der Baumeister selbst nach Vollendung des Baus sterben. Auch das ist ein verbreiteter Zug ${ }^{2}$. Kain stirbt also, nachdem er seinen Bruder geopfert hat, selbst als zweites Bauopfer ${ }^{3}$.

Nach der Analogie der Kainsage haben wir anzunehmen, dab nach der ältesten Fassung der römischen Gründungssage Romulus nicht entrückt, sondern crmordet worden ist. Zwar schildert schon der Dichter ENNIUS die Entrückung. Aber ihr widerstreitet das altertümliche Grab des Romulus, das in neuester. Zeit (1899) wieder aufgefunden worden ist ${ }^{4}$. Nur zur Ermordung stimmt ferner das Fest, das an das Ende des Romulus erinnern soll, die Poplifugia (5. Juli). Die Volksflucht ist das Gegenstück zur Königsflucht (Regifugium, 24. Februar). Ist es hier der Opferkönig, der nach Verrichtung eines Opfers die rituelle Flucht ergreifen mußs, so hat dort das Volk eine Bluttat vollbracht und muß nun flichen. Romulus ist der Sage nach, wenn nicht vom ganzen Volk, so doch von einem Teile des Volkes (den Neubürgern) - oder von den Vertretern des Volkes (den Senatoren) ermordet worden. $\mathrm{Ob}$ auch die Zerstïckelung seiner Leiche ein echter Zug der ursprüng-

I Jos $6_{26}$ I Reg $16_{34}$. E. M^der, Dic Menschenopfer der alten Hebraer, S. I $66 \mathrm{f}$. Zu vergleichen ist - außer den unten Anm. 3 angefuhrten Brudersagen - die Gründungssage von Skutari bei Grimm, Deutsche Mythologic 4 II, S. 957 und bei Fr. Krauss, Das Bauopfer bei den Súdslaven, Mitt. der Anthrop. Gescllschaft in Wien 17, 16: Die Vile forderte als Bauopfer zwei leibliche, gleichnamige Geschivister (Stojan und Stojana).

2 SArtori, Ztschr. f. Ethnol. 30, r6. Krauss, Das Bauopfer, S. 22. Vgl. z. B. Cicero, Tusc. disp. I 47 \&I 4 . Daher das Sprichwort: „Mancher baut ein Haus und muB zuerst hinaus."

2 Ähnlich eine ältere Fassung der Sage von den Baumcister-Brudern Agamedes und Trophonios. Verwandt scheint auch die von J. L. W. Scrrwarz, Der Ursprung der Stammund Grundungssage Roms, S. 44 mitgeteilte sachsische Sage: Zwei Bruder, ein Schmied und ein Steinhauer, bauen eine Höhle. Der Schmied erschlagt den Steinhauer und wird dann selbst in der Hohle lebendig begraben. Ferner die Grundungssage von Plankenwarth (bei Graz): Ludwig crbaut eine Burg, sein Zwillingsbruder Herrmann crschicßt ihn aus Eifersucht und wird dann selbst von den erbitterten Bauern uberfallen und getotet. $M$. UHL.rRz, Schloß Plankenwarth (1916), S. 4 f. Die Forderung cines doppelten Bauopfers liegt uberall zu Grunde.'

* Man half sich über dic Schwierigkeit hinweg mit der Bchauptung, das Grab sei für Romulus bestimmt gewesen, tatsachlich sei aber dort nicht Romulus, sondern Faustulus oder Hostilius beigesetzt worden. Rosenberg in Paulys RE, Art. Romulus Sp. 1101.

s Puutrirch, Qunest. Rom. 63, Rosenberg, Art. Regifugium in Paulys RE. So muB ja auch Kain "unstet und fluchtig" sein (Gen $4{ }_{14}$ ), bis Jahwe die Suhnung (Stigmatisicrung) vollzogen hat. 
lichen Sage ist und kultische Bedeutung I hat, wie SCHWEGLER ${ }^{2}$ vermutet, mag dahingestellt bleiben. Uns muß die Tatsache genügen, daß nach der älteren Fassung der Sage Romulus ebenso wic Kain getötet worden ist ${ }^{3}$.

Es ist merkwürdig, daß auch die Geschichte des Romulus in eine Festlegende ausmündet. Vermutlich hat der römische Erzähler in seiner Quelle bereits den Tod des Stadtgründers mit einem (ausländischen) Feste verknüpft gefunden und hat sich dadurch aufgefordert gesehen, irgendein passendes römisches Fest an dessen Stelle zu setzen.

Die Ermordung des Romulus als ein zweites Bauopfer unmittelbar mit der Stadtgründung zu verbinden, sind wir gewiß berechtigt, obwohl ihm eine lange kriegerische Regierung zugeschrieben wird. Denn wir erhalten von seinen Feldzügen nur dürftige, inhaltsleere Berichte und der Raub der Sabinerinnen ist erst nachträglich (im dritten Jahrhundert) zu Ehren der Sabiner eingefügt worden ${ }^{4}$. „Einzig Romulus Eintritt und Austritt scheint in der ältesten Sage eine Rolle gespielt zu haben" ${ }^{\text {" }}$.

\section{Die Entrückung.}

Hat man sich überzeugt, daß die Entrückung nicht der ursprünglichen Fassung der Romulussage angehört, so überrascht es um so mehr, daß auch sie der vorderasiatischen Urgeschichte entlehnt ist. Über die Entrückung Henochs geht die Genesis mit wenigen Worten hinweg ${ }^{6}$. Wir müssen deshalb die römische Fassung der Entrückungsgeschichte unmittelbar mit der babylonischen vergleichen. Dabei zeigt sich, daß die Entrückung wiederholt ihre Stelle gewechselt hat. . Die Babylonier erzählten sie seit jeher vom Sintfluthelden, sie stand bei ihnen in engem, organischen Zusammenhange mit der Sintflutgeschichte, deren Abschluß sic bildete. Die Quclle des Jahwisten dagegen löste die Entrückung vom Sintfluthelden $a b$ und und übertrug sie auf einen anderen

I RoHde, Psyche I, S. $322 \mathrm{f}$.

2 Römische Geschichte, S. 535.

${ }^{3}$ Dem Rationalismus der neueren Annalisten verdanken wir die Erhaltung der alten Mordgeschichte, aber nicht - wie CARTER (Roschers Lex., Romulus) - glaubt, ihre Erfindung. Rosenberg in Paulys RE (Romulus, Sp. 110r) gibt wenigstons das ausdrücklich $z u$, daß die „alte, vorliterarische Romulussage “ ihren Helden menschlich sterben lieb.

4 Mommsen, Hist. Schr. 122 ff.

s Schwegler aaO. 530 . bemerht.

- Dab diese Darstellung an die Romulussage crinnert, wurde bereits ZAW 35, 9 
Urvater (Henoch). Einmal selbständiz geworden, konnte die Entrückung in jüneren Nachbildungen der Urgeschichte neuerlich den Platz wechseln, um allemal jenen Urvater auszuzeichnen, der für diese Ehre der Würdigste schien, und so war es zuletzt in Rom der Stadigründer, der unter die Gütter versctzt wurde.

Aufmerksame Vergleichung ergibt mit grober Sicherheit, daU die Erzählung von Romulus Entrückung immer noch mit ihrer entferntesten, mittelbaren Quelle, der babylonischen Sintflutsage, in den wesentlichen Zügen übereinstimmt. Unter den babylonischen Fassungen der Sintflutsage lommt für uns vor allem der jüngste Bericht, der des BEROsCs, in Betracht. Sieht man von den Beziehungen zur Sintlut ab, so bleiben etwa folgende Züge übrig:

1. Xisuthros opfert den Göttern.

2. Er verschwindet unmittelbar nach dem Opfer.

3. Die Zurückgebliebenen suchen ihn und rufen seinen Namen!

4. Die Aufklärung wird den Zurückgebliebenen durch die Stimme des Verschwundenen zuteil. Sie befiehlt ihnen, gottesfürchtig zu sein, denn er selbst wohne um seiner Gottesfurcht willen bei den Göttern.

Dazu kommt noch nach dem sumerischen Sintflutberichte: ${ }^{2}$

5. Der Entrückte führt fortan einen neuen Namen.

Alle diese Züge - deren rituelle Bedeutung später untersucht werden soll (unten IV) - kehren in der römischen Sage wieder:

I. Romulus verrichtet ein Opfer, und zwar ebenso wie Xisuthros ein für das ganze Volk dargebrachtes, reinigendes Opfer, eine lustratio des Volkes ${ }^{3}$.

2. Romulus verschwindet wie Xisuthros unmittelbar nach diesem Opfer. Wie Henoch „war er nicht mehr" ${ }^{4}$, denn ein Gott "hatte ihn zu sich genommen"s.

3. Sehnsuchtsvoll rufen die Zurückgebliebenen seinen Namen ${ }^{6}$.

In der Vorlage muß der Zusammenhang des Namenrufens mit einem Festritus deutlich ausgesprochen gewesen sein, denn die Römer stellen wirklich einen solchen Zusammenhang her: PJutarch verweist

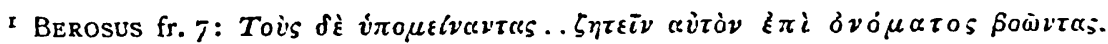

2 LaNdersdorfer, Die sumerischen Parallelen zur biblischen Urgeschichte, S. 13, Jeremias, Das Alte Testament im Lichte des alten Orients ${ }^{3} \mathrm{~S}$. IIg f.

3 Aur. Victor 2, 13. Schwegler, Röm. Geschichte, S. 532.

"Livius I 16: "Nec deinde in terris Romulus fuit."

3 Dion. Hal. II 56: "Y "Y

"Vgl. Ennius, Annales I I I I: „O Romule, Romule dic.." 
auf das Namenrufen bei dem Feste Poplifugia oder Nonae Caprotinaer. Das Volk ruft bei diesem Feste laut verschiedene Namen wie Marcus, Lucius, Cajus, um die damalige Flucht ${ }^{2}$ und die wechselseitigen ängstlichen Zurufe-darzustellen ${ }^{3}$. Der Gedanke liegt nahe, daB der Festbrauch, der vielleicht ursprünglich gar nicht zu den Poplifugia gehört, uns vielmehr daran erinnern soll, daß der neue Name des Entrückten noch uunbekann't ist und man sich vergeblich bemüht, ihn zu erraten 4 Wie wichtig die Kenntnis des richtigen Namens eines Gottes für dessen Kultus ist, ist bekannt ${ }^{5}$.

4. Nun läßt sich, wie in der Xisuthros-Sage die Stimme des Verschwundenen vernehmen ${ }^{6}$. Romulus verkündet, daß er ein Gott geworden sei, und läßt es auch nicht an weiser Ermahnung fehlen ${ }^{7}$.

5. Zugleich verkündet Romulus seinen neuen Namen Quirinus ${ }^{8}$

Die genaue Übereinstimmung so vieler und eigenartiger Züge kann kein Zufall sein. -Daß aber jeder Zusammenhang mit der Sintflut fehlt, spricht nicht gegen die Entlehnung, sondern -dafür. 'Denn wir wissen ja bereits, daß die Römer nicht unmittelbar aus der babylonischen Quelle, sondern - und auch das nur. mittelbar - aus einer der Genesis nahestehenden. Umarbeitung geschöpft haben. Und da war die Entrückungsgeschichte bereits von dem Sintfluthelden auf einen anderen Urvater (Henoch) übértragen.

Was sonst noch von der Entrückung des Romulus erzählt wird, ist nebensächliche Ausschmückung, kann aber immerhin der für uns verlorenen Umarbeitung - also der Henochságe - entlehnt sein. Mancher Zug erinnert an Elias. So, daß Romulus in einem Gewitter entschwindet ${ }^{9}$, daß er im Wagen seines göttlichen Vaters'gen Himmel fährt ${ }^{\mathrm{IO}}$, daß er sich in feurigen Waffen zeigt ${ }^{\mathrm{II}}$, und zwar einem

I Zwei verschiedene Feste, die Plutarch vermengt.

2 Oben bei S. 70, Anm. 5 .

3 So Plutarch, Romulus 29.

4 Vgl. das Märchen vom Rumpelstilzchen.

3 Vgl. z. B. Gunkel, Komm. zu Gen $322_{20}$

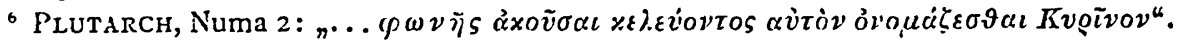

' Ovid, Fasten Il 508: „patrias artes militiamque colant.." Vgl. Berosus fr. 7:

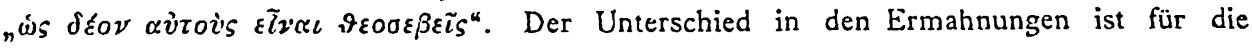
beiden Völker, kennzeichnend:

B Quirinus hat ursprünglich mit Romulus gewiß nichts zu schaffen. Die von auswärts übernommene Sage knüpft hier wie überall an bereits Vorhandenes an.

- Livius I 16: "subito coorta tempestas cum magno fragore tonitribusque".

10 Ovid, Fast. II 496: "rex patriis astra petebat equis". 'Vgl. auch Metam. XIV 820-824.

II Plutarch, Romulus 28. 
Einzelnen, dem Proculus, den er eben dadurch zu seinem Nachfolger bestimmt ${ }^{x}$.

Romulus wäre also Henoch. Aber Romulus kann nicht Henoch sein, wenn er Kain ist. Hat die ursprüngliche Fassung der Sage (vor EnNius). an der Ermordung des Stadtgründers festgehalten, so muß sie - ganz wie die Genesis - die Entrückung von einem andern Könige erzählt haben ${ }^{2}$. Proculus muß der Nachfolger dieses Königs gewesen sein. Nun erscheint ein König Proca in der Königsliste von Alba Longa. Das ist ein aus verschiedenartigen Stoffen zusammengestoppeltes, spätes Machwerk. Es kann immerhin einzelne Gestalten aus einer älteren römischen Königsliste oder unmittelbar aus der Geschichte der Urväter (Aboriginer) übernommen haben. Proca ist Proculus; die Verschiedenheit der Endung ist ohne Bedeutung. Dieser König ist nun in der albanischen Liste deŕ Nachfolger eines Königs Aventinus und von Aventinus erzählt uns Augustin wirklich, daß er entrückt und als Gott verehrt worden ist ${ }^{3}$. Wenn Aventinus von andern Schriftstellern als König der Aboriginer bezeichnet wird ${ }^{4}$, so ist das vielleicht ein Hinweis darauf, daß wir es auch hier ursprünglich mit einem der Urväter zu tun haben.

In der offiziellen römischen Königsliste fehlen Aventinus und Proculus. Die Entrückung ist auf Romulus übertragen. Im übrigen -scheint Numa $^{5}$ die - freilich für uns schwer faßbaren - Züge des entrückten Urvaters übernommen zu haben:

Henoch wandelte vor 'Gott (Gen- $5_{22}$ und ${ }_{24}$ ). Das heißt nach GUNKEL: ${ }^{6}$ "antik gesprochen: ihm ist Gott erschienen und hat ihm

I Plutarch, Numa 5: Die Romer wollten den Proculus zum Könige wählen. Daß die Sabiner diese Wahl vereitelten, ist (wie alles Sabinische in der Kónigsgeschichte) auf Rechnung einer Umarbeitung im 3. Jahrhunderte zu stellen.

$2 \mathrm{Da}$ Henoch beim Jahwisten der Nachfolger des Stadtgrunders ist, wurde Numa als des Romulus Nachfolger am nachsten liegen. Wirklich ist die Vergotterung des Numa bereits, behauptet worden, und zwar von PaIS, Storia di Roma I I, 29I. Was De Sanctis, Storia dei Romani 1359 einwendet, schlaggt nicht durch. Aber es handelt sich um eine Flußentrúckung (Rohde, Psyche II 377, Anm. 2), die fur uns schwerlich in Betracht kommt.

- Civ. dei 18, 21: "Alii sane noluerunt eum in proelio scribere occisum sed non comparuisse dixerunt: sed nec ex eius vocabulo appellatum montem, sed ex adventu avium dictum Aventinum." Vgl. Varro, de 1. 1. V 43 und Servius zu Aneis 7, 657. Danach scheint NaEvius die (mittelbare) Quelle des Kirchenvaters zu sein.

4 Servius aaO.

$s$ Vgl. oben Anm. 2.

'Handkommentar' ${ }^{3}$ S. 136. 
Geheimnisse offenbart." Numa erhält Offenbarungen im persönlichen Verkehre mit der Göttin Egeria, mit Tacita und anderen Musen, mit Picus und Faunus und schließlich mit Jupiter selbst. DaB sich die Offenbarung Jupiters auf ein Menschenopfer bezieht ${ }^{\mathrm{I}}$, erinnert uns wieder an den Ideenkreis, der die ganze Urgeschichte beherrscht. Das babylonische Urbild Henochs, Enmeduranki ${ }^{2}$, ist der Stifter eines wichtigen babylonischen Priestertums. Die Bedeutung Numas liegt gerade darin, daB alle wichtigeren Priestertümer auf ihn zurückgefübrt werden. Henoch ist (nach Gen $5_{23}$ ) $36_{5}$ Jahre alt geworden: das ist die Zahl der Tage des Sonnenjahres ${ }^{3}$. Gerade dem Könige Numa wird die Einführung des Sonnenjahres von 365 Tagen zugeschrieben ${ }^{4}$. Andere Beziehungen aufzusuchen, verbietet die Dürftigkeit der biblischen Überlieferung ${ }^{5}$.

Auf Numa folgt der vom Blitze erschlagene .König Tullus Hostilius ${ }^{6}$. Gehört auch dieser zu den Urvätern, so ist man versucht, an Mehujael, den von Gott vertilgten, zu denken ?

\section{Das Sintflutfest.}

Kaleidoskopartig wechseln die Bilder in der Urgeschichte. Die Urväter ändern die Reihenfolge, einige fließen zusammen, andere verdoppeln sich, mit Leichtigkeit tauschen sie einzelne Züge aus. Die Entrückung ist von dem babylonischen Sintfluthelden auf Henoch und von diesem in Rom auf den Stadtgründer übertragen worden ${ }^{8}$.

I Plutarch, Numa 15. Schwenn, Die Menschenopfer bei den Griechen und Rómern $(1915)$ S. 177.

2 ZINLMERN, Die Keilinschriften ${ }^{3} j 33 \mathrm{f}$. O. Weber, Literatur der Babylonier $\S 53$.

2 ZnMmers aaO. 540.

4 Plutarch, Numa 18, Schwegler aaO. 545, Anm. 3.

s Gehört es hierher, daß Numa gerade an dem Tage geboren worden ist, an dem Romulus die Stadt eroaute? Plutarch, Numa 3, Schwegler aaO. 558 vgl. mit Budde, Bibl. Urgeschichte, S. I2I ( $\mathrm{zu}$ Gen $4_{17}$ ). Ist bei den vergrabenen Schriften Numas (SChwegler, S. j64) an die in Sippar vergrabenen Schriften zu denken (Bóklen, Arch. Rel. Wiss. $6,3_{4}$ ff., MAder, Menschenopfer der alten Hebraer, S. 49)?

- In der Königsliste von Alba Longa heißt der vom Blitze erschlagene Romulus oder Remulus oder (nach der Konjektur von DE SANCTIS I 205) Amulius. Uber die Bedeutung des Blitztodes vgl. RoHde, Psyche I $320 \mathrm{ff}$. und dazu Cicero. bei Augustin, De cir. dei 3, 15 . ' BuDDE, Urgeschichte, S. 128.

8 Dafür hat vielleicht Noah rom Landmanne (Gen 720 ), d. h. vom Stadtgrunder den Weinbau an sich gezogen. Eine griechische Sage scheint den Stadtgründer Oineus, Weinmann, zu nennen. Ihm stellt sic statt des Hirten den Jager - Toxeus, den Bogenmann - gegenüber. APOLLODOR I 8, ZAW 35, 5, Anm. 4. War Kain in irgendeiner Lesart der Urgeschichte der trunkene Weinbauer, so war dort vielleicht Japhet sein, nicht 
Ihre ursprüngliche Bedcutung läßt sich vielleicht erraten, wenn wir sie wieder in den alten Zusammenhang stellen. Sie gehört zur Sintflut. Schon die alten sumerischen Berichte wissen, daß die Götter dem Sintfluthelden "Leben wie einem Gotte gegeben" und daß sie ihn in ein fernes Land versetzt haben ${ }^{\mathrm{r}}$.

Die Sintfluterzählung war ursprünglich eine Kultlegende. Dafür spricht die Umständlichkeit der cinzelnen Angaben z. B. über die Abmessungen der Arche, über die Zahl der aufzunehmenden Tiere, über die Kalenderdaten der Flut und Ähnliches. Diese Angaben sind einst bei den Babyloniern, bei den Syrern, ja selbst noch bei den Griechen rituelle Vorschriften gewesen. Drum steht, nach der treffenden Bemerkung GRUPPEs ${ }^{2}$, der Sintflutmythos "als Ritualmythos von universalem Charakter in der griechischen Sagenwelt einzig da. Daß er ursprünglich die Einführung eines Rituals zu erklären oder zu begründen bestimmt, also eine Legende war, kann, da der Zusammenhang mit dem Gottesdienst an mehreren Stellen durchscheint, in Athen und Delphi aber deutlich erkennbar ist, nicht wohl bezweifelt werden ..."

Ein großes Sühnfest, das dereinst irgendwo in Vorderasien zuerst gefeiert worden ist, hat die Aufmerksamkeit fremder Völker erregt, es ist nachgeahmt worden und mit ihm ist die Festlegende von Volk zu Volk gewandert. Nur ist die Legende, dauerhafter und beweglicher als das Fest, weiter vorgedrungen' und länger in der Erinnerung bewahrt worden. Schon im Gilgameschepos und beim Jahwisten ist sie in einer Weise ausgeschmückt und verändert, die erkennen läßt, daß sie dort der engen Beziehung zu einem einheimischen Kultus bereits entbehrte.

Dennoch läst sich jenes alte Sintflutfest wenigstens in den wichtigsten Zügen wiederherstellen. Ess galt der Reinigung des Landes und der Beruhigung des göttlichen Zornes. Die Reinigung erfolgte durch Wasser. LUCIAN $^{3}$ beschreibt sie in seiner Darstellung des Sintflutfestes

Noahs Sohn, also eine Person mit Henoch, dem Eponymen der ersten Stadt. Und dann war diese Stadt nach eben derselben ortlichen Fassung der Grundungssage gewiß keine andere als Japho, d. i. " Joppe Phoenicum, antiquior terrarum inundatione, ut ferunt ${ }^{*}$ (Plinius, Nat. Hist. 5, 14). Dann war aber Japhet ein Vorfahre des Sintluthelden genau so wie in der griechischen Sage Japetos: Vielleicht ist Japetos als ein anderer Henoch von Kronos entruckt worden und wohnt deshalb bei ihm nach llias 8, 479 - freilich im Tartaros. Der Name Kronos bežeichnet haufig semitische Gottheiten (so z. B. in der Sintflutgeschichte des BEROSUs).

I Jeremias, Das Alte Testament ${ }^{3}$ I 20, LANDERsDorfer, Die sumerischen Parallelen, S. I3.

2 Griechische Mythologie I 446.

3 De Syria dea 13 (vgl, auch 48 ). 
von. Hierapolis. Gemäß einer Anordnung Deukalions brachte man Meerwasser ${ }^{\mathrm{I}}$ in den Tempel, und zwar taten dies nicht'nur die Priester, sondern Pilger aus ganz Syrien, aus Arabien und aus Babylonien. Das Wasser gossen sie im Tempel aus und es floß dort durch eben die Spalte, ab, durch die einst das Wasser der deukalionischen Flut abgeflossen ist. Die gleiche Erdspalte wurde auch in Athen gezeigt und auch dort wurde, wie es scheint, ein ähnliches Fest gefeiert, die Hydrophorien ${ }^{2}$.

Nicht erweislich, aber doch recht naheliegend ist die Vermutung, daB die Erde, während der heilige Ort vom Meerwasser überströmt wurde, kraft einer Fiktion als überschwemmt galt und daß deshalb die Opfertiere in einem nach besonderer Vorschrift erbauten Schiffswagen ${ }^{3}$ über das trockene Land zum Heiligtume gesteuert wurden. Solche Schiffsumzüge sind im Kultus weit verbreitet ${ }^{4}$.

Da die Sünden des ganzen Volkes oder der ganzen Menschheit gesühnt werden sollten, nahm man Opfertiere jeder Art in die Arche auf. An die unreinen Tiere hat die alte Legende schwerlich gedacht ${ }^{5}$. Erst durch ihre Einbeziehung ist der ganze Vorgang abenteuerlich und unausführbar geworden.

Zur Beruhigung des Herzens der erzürnten Götter wurden die Tiere verbrannt. "Noah" erzählt der Jahwist $\left(8_{20}\right)$ nbaute Jahwe einen Altar und nahm von allen reinen Tieren und brachte Ganzopfer dar auf dem Altar." Eine weithin leuchtende Flamme verzehrte das ungeheure Opfer, das an manchen Orten „Fackel" oder ähnlich hieß ${ }^{6}$.

I Meerwasser! Es handelte sich also nicht um Wasserspenden für die durstigen Seelen der Sintflutopfer, sondern um eine kultische Reinigung. Rohde, Psyche II 405. J. SCheftelowitz, Arch. Rel. Wiss. 17, 397. Auch die Sintllut selbst wird zuweilen als eine Abwaschung aufgefaBt. Jeremias, Das Alte Testament ${ }^{3}$ S. 432, Usener, Sintflutsagen, S. 27. Aber die Sintflut sollte die Erde nicht bloß von den Sünden, sondern von den Sundern selbst befreien. Erst $\mathrm{nach}$ der Sintflut - soewill es die Legende $\left(\operatorname{Gen} 8_{2 \mathrm{r}}\right.$ ) - haben die Götter auf die Vertilgung der Sünder verzichtet.

2 Plutarch, Sulla I4. Rohde, Psyche I 238.

3 Der carrus navalis, von dem manche den Namen Karneval ableiten wollen.

4 Ein ägyptisches Schiff, das von vierzig Priestern getragen wird, zeigt die Abbildung bei JerexIIAS aaO.385, ein attisches auf Rädern die Abbildung bei ÚsENER aaO. I 17. Schiffsumzūge finden sich beim Auszuge des Marduk (Zimmern aaO. 515), bei der Feier der Panathenäen (USENER aaO. 125 f.) und sonst häufig. Auch das deutsche Narrenschiff gehört hierher. Grimm, Myth. I 213, Mannhardt, Wald- und Feldkulte I $592 \mathrm{ff}$.

5 Nur von den Tieren des Feldes, die Grünes fressen, spricht der Tex́t bei Jeremias, S. 125. vgl. hieza Znmern aaO. 548, Anm. 4.

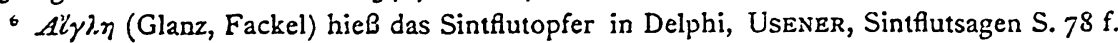
Bei dem Feste $\pi v \rho \eta$ oder lacutcis in Hierapolis ist der Zusammenhang mit der Sintflut nicht klar, unten S. 78, Anm. I. 
Vielleicht wurde auch die Arche selbst verbrannt. Sie bestand aus einem besonderen, gut brennenden Holze $\left(G e n 6_{14}\right.$ ) und war nach dem Gilgamesch-Epos reichlich mit Brennstoffen (Erdpech, Asphalt, Öl) beladen. Die Tiere wurden vermutlich in einer bestimmten Reihenfolge aus der Arche genommen, geschlachtet und ins Feuer geworfen ${ }^{x}$. In dieser Weise wurde nach PAUSANIAS das große Ganzopfer im messenischen Heiligtume der Kureten ${ }^{2}$ verrichtet: man begann mit den Rindern und Ziegen ${ }^{3}$.und ging bis zu den Vögeln herab. Eine bestimmte Ordnung scheint noch die Priesterschrift (Gen $8_{19}$ ) im Auge $\mathrm{zu}$ haben: die Tiere gingen "nach ihren Gattungen" aus der Arche.

" Das große Opfer beruhigt die erzürnten Götter (Gen $8_{2 I}$ ). Es ist so sehr der Kern der Erzählung, da $B$ es ihrem Helden den Namen gegeben hat. "Noah" ist das babylonische nuhbu, der technische Ausdruck für das Beruhigen der erzürnten Gottheit ${ }^{4}$. Wirklich feierten die Babylonier ein Nuhhu-Fest. Ihr Kalender kennt einen "Tag der Beruhigung des Herzens" (um nuh libbi)s.

Nun zur Entrücḳung. Wo Menschenopfer üblich waren, da gehörte zu den reinen, d. h. zum Opfer geeigneten Tieren auch, als kostbarstes, der Mensch. Die Legende mußte also auf die Frage Rede stehen, warum bei dem großen Beruhigungsfeste nicht auch Menschen geopfert wurden ${ }^{6}$. Sie beantwortet die Frage in der Art, in der sie

I Bei dem Fackelfeste in Hierapolis sollen sie lebendig verbrannt worden sein. LucinN aaO. 49. CleneN in der-Festschrift für Baudissin S. 104.

2 Sind hier unter den Kureten die Urvater verstanden, wie bei Drodor 5,65 und bei Justin 44,4 ? Bei Justin wollen übrigens die Herausgeber durchaus "Cunetes" fur Curetes lesen, als wàre die (in den außersten Westen verlegte) Urgeschichte eine bei einem bestimmten Volke in Spanien heimische Sage.

- Der sumerische Sintflutheld opfert einen Ochsen, dann ein Schaf... die Fortsetzung ist nicht erkennbar. JEREMIAS, S. I I g.

+ Zimmern, Die Keilinschriften ${ }^{3}$ S. 610. Auf die richtige Deutung des Namens Noah hat die LXX gefuhrt: ${ }_{n} \delta \iota \alpha \nu \alpha \pi \alpha v \sigma \varepsilon \iota^{\mu}$. Nach LANGDON soll schon der sumerische Sintflutheld "Beruhigung (Tagtug) geheißen haben. LANDERSDORFER, Die sumerischen Parallelen, S. 50. Dagegen aber zweifelnd Jeremias, Altes Testament ${ }^{3} \mathrm{~S}$. I 18 und sehr entschieden UNGNAD, ZDMG 7r, $254 \mathrm{f}$. Ob in der griechischen Flutlegende von der „Beruhigung " des Zeus die Rede war, ist ungewiß. Die von PaLmerius vorgeschlagene Lesart xõ $\Delta \iota \grave{s} \pi \alpha v o \mu \varepsilon v o v$ im Sintflutberichte des Marmor Parium ( neueren Herausgebern verworfen.

5 Zimmern aaO. S. 592.

- Von einem Menschenopfer erzählt keine Fassung der Sintflutsage. Eigentumlich ist nur, daß (bei Strabo p. 435) zwei zur Phthiotis gehörende Inselchen Deukalion und Pyrrha heißen. Sind Deukalion und Pyrrha nach einer einheimischen (griechischen) Sage anlaßlich einer Úberschwemmung als Menschenopfer ins Meer gesturzt und dann in Klippen 
auch sonst das Abkommen von Menschenopfern darzustellen pflegt: die zum Opfer bestimmten Menschen wurden einst von den Göttern selbst durch Entrückung gerettet ${ }^{\mathrm{I}}$.

Die Art, wie die babylonische Erzählung die Entrückung darstellt, deutet auf einen bestimmten Ritus. Es scheint, daß man. Menschen in die Arche einschloß, aber rechtzeitig entkommen ließ. Dann suchte man sie und rief sie beim Namen ${ }^{2}$, bis eine Stimme - vielleicht aus dem Innern des Heiligtums ${ }^{3}$ - verkündete, daß die Götter selbst jene Menschen entrückt haben und daß ihr Zorn beruhigt sei.

\section{Die ersten Menschen.}

Bei den Römern hat sich nur ein Teil der asiatischen Urgeschichte dauernd erhalten. Vollständig wurde sie von den Griechen bewahrt. In Arkadien beginnt die Reihe der Urkönige mit. Pelasgus. Das ist der erste Mensch, denn er ist, wie ein alter Dichter ${ }^{4}$ singt, der schwarzen

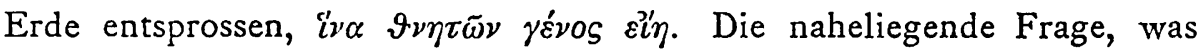
für Menschen denn dieser Urkönig eigentlich beherrscht habe, begegnet schon bei PAUSANIAS. Mit eben diesem Pelasgus beginnt bereits die Reihe der Erfinder: er lehrt die Menschen sich von Baumfrüchten nähren und verfertigt die ersten Kleider. Darin erinnert er an Adam. Aber von der Ehe ist in der arkadischen Sage keine Rede und sie gehört denn auch aller Wahrscheinlichkeit nach gar nicht an diese Stelle der Ur-

verwandelt worden? Eine Geschichte dieser Art erzählen - merkwürdig genug - die Zuni-Indianer: ${ }_{n} \mathrm{Da}$ die Fluten die Hochflache der Masa erreichten, so war ein Menschenopfer zu ihrer Beschwichtigung nötig. Ein Jüngling und ein Mädchen, die Kinder zweier Priester, wurden in das Wasser geworfen, dort aber wurden sie in Stein verwandelt, in zwei große Felsen, die Vater und.Mutter heißen. Beiden wird geopfert.“ Gerhard, Der Mythus von der Sintflut (1912), S. 99 f.

I So hat Artemis nach dem Berichte der Kypria die zum Opfer bestimmte Iphigenie entrūckt, nach Tauris versetzt und unsterblich gemacht; auf die Ähnlichkeit der Erzahlung von der Entrückung Henochs weist ROHDE, Psyche I 85, Anm.' I ausdrücklich hin. Dem gleichen Muster folgt die Sage von der Entrückung des Phrixos. Auch das Abkommen des menschlichen Bauopfers wird durch eine Entrückungsgeschichte erklart: Grimm Myth. III 320. In der Folge werden statt der Menschenopfer bloß die Beigaben (Gefaße für Speise und Trank, Lampen) oder leere Särge eingegraben. SARToRI, Z. f. Ethnol. 30 50 ff. Gressmans, Art. Menschenopfer in RGG, Heberdey Arch. Rel. W. 17, 678.

2 Darin liegt eine besondere Ehrung der Entrückten, vgl. RoHDE, Psyche I, S. 174 Anm. I und Gen 4'ss.

3 Vgl. Lucian, De Syria dea 10: Aus dem verschlossenen Tempel ließen sich ofters ror vielen Zuhörern Stimmen vernehmen.

4 Asios bei Pausanias Vill $\mathrm{I}$. 
geschichte. Die erste Ehe ist überall eine göttliche Ehe gewesen; es hängt mit.dem israelitischen Monotheismus zusammen, daB die Einsetzung der Ehe in der Genesis aus der Göttergeschichte in die Menschengeschichte herabgerückt und mit der Urvätersage verquickt worden ist I . Bei den Babyloniern ist die heilige Ehe die Ehe zwischen Marduk und Sarpanitu oder Istar ${ }^{2}$, bei den Ägyptern ist es die Ehe zwischen Osiris und Isis, bei den Griechen die Ehe zwischen Zeus und Hera.

Aber trotz ihrer Vermenschlichung hat die Ehe zwischen Adam und Eva ihre vorbildliche Bedeutung als heilige Ehe bewahrt. Sie bietet uns merkwürdige Aufschlüsse über ein altes, vorisraelitisches Eherecht. Zwei Verbote treten scharf hervor: das der Exogamie und das der Kinderehe. Zuerst wird uns die Notwendigkeit der Blutsverwandtschaft zwischen Mann und Weib eingeschärft. Was nämlich heute AnstoB erregt, die Geschwisterehen der Kinder des ersten Paares, das war ohne Zweifel beabsichtigt. Bei anderen Völkern ist die heilige Ehe selbst eine Geschwisterehe: Osiris und Isis, Zeus und Hera waren Geschwister. Dafür machte damals eine Frage Schwierigkeiten, die uns heute nicht das geringste Kopfzerbrechen verursachen würde: Wenn Gott wirklich Mann und Weib unabhängig voneinander geschaffen hätte (vgl. Gen I $_{27}$ ), dann wären sie miteinander nicht verwandt gewesen. Die erste Ehe wäre nicht nur keine Geschwisterehe, sie wäre überhaupt keine Verwandtenehe gewesen. Die naive Erzählung Gen $2_{18-20}$ zeigt, daß Jahwe an einer sol̄chen Ehe keinen Anstoß genommen hätte, aber Adams königlicher Sinn weist die ihm angebotenen fremden Wesen zurück. Er will - das ergibt sich klar aus dem Folgenden - nur eine Blutsverwandte zum Weibe nehmen. Es gelingt Jabwe, die merkwürdige Schwierigkeit in der bekànnten Weise zu überwinden, und sofort begrüBt Adam die auf wunderbare Weise geborene Tochter mit den Worten: „Diese endlich ist Bein von meinem Bein und Fleisch von meinem Fleisch..." ${ }^{3}$. Die erste Ehe ist also die allerengste Verwandtenehe, nicht eine Geschwisterehe wie bei den Griechen und Ägyptern, sondern eine Ehe zwischen Vater 'und Tochter wie bei den Babyloniern und Indern. Die Blutschande erregt keinen AnstoB, im Gegenteile, sie ist, vornehmer Brauch. Der Stammbaum eines edlen Geschlechtes kann nicht besser angefangen werden.

\footnotetext{
I'Vgl. LANDERSDORFER, Die sumerischen Parallelen, S. 92. $\quad{ }^{2}$ ZIMMiern aaO. 37 I, 375.

${ }^{3}$ Das ist die unzweideutige Bezeichnung der Blutsverwandtschaft, vgl. z. B. Gen $29_{\mathrm{x}}$ * Ähnlich im Punischen nach Servius zu Aeneid. 4, 625.
} 
In- der sumerischen Paradieșeserzählung ${ }^{x}$ ist die Göttin Ninella zugleich die Tochter und die Gemahlin des Gottes Enki². Von Herakles erzählt Megasthenes (fr. 23), daß er für seine Tochter Pandaea keinen würdigen Eidam gefunden und sich deshalb selbst mit ihr vereinigt habe, um das Geschlecht der indischen Könige zu erzeugen ${ }^{3}$. Nach indischen Quellen hat Manu seine auf wunderbare Weise geborene Tochter Ida zum Weibe genommen und ist so zum Stammvater der Dynastie (nicht der ganzen Bevölkerung) geworden ${ }^{4}$. Daß das Weib seine Tochter ist, stellt Manu ausdrücklich fest ${ }^{5}$, dann erst verlangt er, mit ihr Nachkommen zu erzeugen. Nach dem Berichte der Genesis stammen die Völker Ammon und Moab aus einer solchen Verbindung.

Die heilige Ehe ist das Vorbild für die Ehen der Menschen. Aber es kann nicht - auch bei den Ägyptern ${ }^{6}$ nicht - allgemeine Sitte gewesen sein, die eigene Schwester oder gar die eigene Tochter zu heiraten. Wahrscheinlich begründete die Ehe selbst, unter entsprechenden Bräuchen abgeschlossen, eine künstliche Blutsverwandtschaft: sie war mit einer Verschwisterung oder einer Adoption verbunden, um die heilige Ehe nachzubilden und zugleich die rechtliche Stellung der Frau z. B. hinsichtlich des Erbrechtes zu bestimmen?.

Auf einen die Blutsverwandtschaft begründenden Hochzeitsbrauch ${ }^{8}$ scheint der dunkle Vers Gen $2_{24}$ anzuspielen, in dem man eine

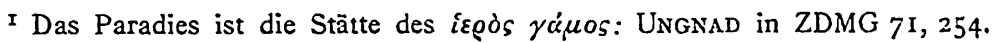

2 LANDERSDORFER aaO. 42.

3 Zugleich behandelt diese merkwürdige Legende ebenso wie die biblische die Frage der Kinderehe: Pandaea war erst sieben Jahre alt, aber Herakles machte sie mannbar.

4 Gargiter, Journal of the Royal Asiatic Society 1914, 269.

5 "Wer bist du? " Deine Tochter." "Wieso, Herrliche, meine Tochter?" "Aus jenen Opfergaben hast du mich erzeugt usf." Usener, Sintflutsagen, S. 27, Jeremias, Das Alte Testament ${ }^{3}$ S. 132. Die Analogie mit dem Verhalten Adams fallt auf. Hier und dort wird es sich um ein Stück eines alten Hochzeitsritus handeln. Vgl. unten Anm. 8.

- Die Ägypter hielten sich nach DrodoR I 27 an das Vorbild der heiligen Geschwisterehe. Vgl. E. WeIss, Endogamie und Exogamie im römischen Kaiserreich, Ztschr. f. RG. rom. Abt. 29, 340. Aber die Papyri lassen erkennen, daß auch Ehegatten, die sich - wie üblich - als Geschwister bezeichneten, von verschiedenen Eltern stammten, vgl. z. B. Helbisg, Auswahl aus griechischen Papyri S. 64 , und so kann es sich selbst in Fallen verhalten, wo sich die Ehegatten ausdrücklich als vollbürtige Geschwister bezeichnen (E. WeIss aaO. 35 I ff.): die Eltern des Mannes hatten eben die Schwiegertochter adoptiert.

7 Bei den Rümern war die Frau an Tochterstatt - filiae loco. Garus, Instit. 3, 3.

- Von einem die Zeugung nachbildenden Opfer ist in der Manulegende die Rede. Hierher gehort auch, daB sich bei'den Indern Braut und Brautigam gegenseitig mit ihrem Blute bezeichnen. Westermarck, Geschichte der menschlichen Ehe, S. 422. Auch die 
Erinncrung an das Mutterrecht oder gar eine Vorabnung der platonischen Philosophic hat finden wollen: „Darum läßt der Mann Vater und Mutter und hängt dem Weibe an, so daß sie ein Fleisch werden" - ein Fleisch, d. h. (wie in v. 23): blutsverwandt. Der Freier hängt dem fremden Weibe an, nicht etwa dauernd, sondern so lange, bis sie durch die rituelle Ehe seine Blutsverwandte geworden ist. Dann erst darf er sie heimführen.

Die Erzählung von Adam und Eva hat noch eine zweite Aufgabe. Auffallend tritt als ein wichtiges Ereignis das Erwachen des Schamgefühls hervor. Das ist ja der einzige Erfolg, den die ersten Menschen durch das Essen der verbotenen Früchte erreichen. Es taten sich ihnen beiden die Augen auf und sie erkannten, daß sie nackt waren; so nähten sie Feigenblätter zusammen und machten sich Schürzen daraus. Der Zusammenhang mit der Ehe ist leicht $z u$ erraten. Die Legende lehnt die weitverbreitete $\mathrm{K}$ inderehe ab. Die Heiratenden müssen reif sein. Die Reife wird aber nicht durch körperliche Besichtigung festgestellt, auch nicht an ein bestimmtes Alter gebunden, vielmehr entscheidet das Erwachen des Schamgefühles. So war es bei den Indern ${ }^{\mathrm{I}}$ und ursprünglich auch bei den Griechen. ${ }^{2}$ Deshalb gehört zum Hochzeitsritus die Verschleierung der Braut.

\section{Die Urväter und der Festkalender.}

Überblickt man den Verlauf der Urgeschichte, so erhält man den Eindruck, daß sie aus einer Kette von Festlegenden besteht. Drei von den Urvätern (Henoch, Jered, Noah) tragen geradezu den Namen von

Namensubertragung (Gen $2_{23}$ ) kann Adoptionsritus sein, vgl. Gen $48_{10}$. Die romische Braut (oben Seite 81, Note 7) antwortet auf die Frage des Brautigams, wie sie heiße: Quando (oder ubi) tu Gaius ego Gaia. Plutarcir, Quacst. Rom. 30.

× Vgl. Jaiminigrilhyasutra bei BirnNDARKaR, History of child-mariage in der Ztsch der deutschen morgenlandischen Gesellschaft 47, 154: ${ }_{n}$ Er soll cin Weib sciner eigenen Kaste heiraten, das keine nagnikâ ist ${ }^{u}$. Der indische Kommentar bemerkt hiezu: ${ }_{n}$ Keine nagnikâ d. h. in dem Alter, worin sic aus Schamhaftigkeit cin Kleidungsstuck freiwillig tragt".

.2 Denn nur so erklart sich die verworrene Hochzeitsgeschichte bei Pausanias III 20, 10. Odysseus hat die Penelope geheiratet, aber ihr Vater will sic nicht ziehen lassen. Warum? Das Folgende zeigt: weil sie sich noch nicht schimt, d. h. weil sic noch zu jung ist. Odysseus entfuhrt sie, Ikarius setzt dem Paare nach. Odysseus stellt 'der Gattin nun frei, zu entscheiden, ob sic bei ihm bleiben oder dem Vater folgen wolle. Statt aller Antwort verhüllt sich Penelope. Nun gibt sich merkwürdigerweise Ikarius zufrieden und errichtet eine Bildsaiule der $\mathrm{Scham}$. Vielleicht gehort auch der romische Ausdruck "vesticeps ${ }^{\text {" }}$ fur pubes in dicsen Zusammenhang. 
Festen. - Die Reihenfolge der Könige muß also wohl ursprünglich dem Kalender entsprochen haben. Es besteht kein Grund, diese Reihe mit Noah, dem zehnten Könige, zu schließen. BERosus kennt $z$ wölf Urkönige. Zwei von ihnen haben nach der Flut gelebt ${ }^{x}$. Jeder Urkönig entspricht also einem Monate. Da der Festkalender von Zeit zu Zeit und von Volk zu Volk wechselt, so hat die Ordnung der Urkönige mannigfaltige Umstellungen schon zu einer Zeit erfahren, da der Zusammenhang mit den Festen noch klar war. Mancher von ihnen ist in der Genesis wohl ganz ausgemerzt worden. Dafür sind dann andere verdoppelt worden. Enoš und Adam, Šet und Kain (Kenan) sind vielleicht dieselben Personen. Die ursprüngliche Reihenfolge herzustellen, wäre ein aussichtsloses Beginnen. Jedenfalls aber tritt die Beziehung zum Kultus überall mehr oder minder deutlich hervor.

r. Der "Mensch" (Adam, Eno§) gehört jedenfalls an die Spitze, also, wenn unsere Vermutung richtig ist, zum Neujahrsfeste. War dieses zugleich das Hochzeitsfest des Sonnengottes ${ }^{2}$, so lag es besonders nahe, die Hochzeitslegende (Verbindung mit Eva) auf den ersten Urvater zu übertragen. Bei Enoš ist wenigstens die Beziehung zum Kultus offenkundig. Von ihm wird ja berichtet, daß er der erste war, der den Namen Jahwe anrief (Gen $4_{26}$ ). Damit ist nicht etwa die Stiftung des besondern israelitischen, sondern die des' Gottesdienstes überhaupt gemeint ${ }^{3}$.

2. Der Stadtgründer (Kain) ist der zweite Urkönig nicht nur beim Jahwisten, sondern auch in der griechischen Sage ${ }^{4}$. Dazu stimmt, daß der zweite Monat der der Tempelgründung ist ${ }^{5}$.

3. Bei Henoch weist schon der Name auf ein Fest der Einweihung hin. Dieser Urvater steht bein Jahwisten schwerlich an der richtigen Stelle. Wahrscheinlich hat der Jahwist an die Einweihung der ersten Stadt gedacht, die freilich nicht gut bis zum siebenten Geschlechte hinausgeschoben werden konnte. Dem Kalender folgt die Priesterschrift.

I Euechoos und Chomasbelos, Berosus fr. II.

2 ZnMmerN aaO. $37 \mathrm{I}$.

3 Was kann er geopfert haben, da doch das Opfer der Feldfrüchte auf Kain, das Tieropfer auf Abel und das Menschenopfer wieder auf Kain zurückgeführt wird? Vielleicht wilden Honig. Bei den Griechen ist es der kretische Urkönig Melisseus - also der Bienenmann -, der zuerst den Göttern geopfert hat (Didymus bei Lacrantius, Inst. I 22) und der erste Urkönig der Kureten Gergoris - vielleicht eine Person mit Melisseus soll das Honigsammelń erfunden haben (Justis 44, 4).

4 Bei den Arkadern: Pausanias VIII 2; in der Kuretensage: Justin 44, 4. Die sieben Stādte des Kureten Habis erinnern an die sieben vorsintflutlichen Stadte der Babylonier.

s ZimMern aaO. 74, 331. I Reg $6_{1}$ Esr 36. 
Der siebente babylonische Monat heị̂t Tašrit, d. h. Einweihung ${ }^{1}$. Der salomonische Tempel, der im zweiten Monate gegründet worden ist, ist im siebenten Monate eingeweiht worden (I Reg $8_{2}$ ). Auch der entsprechende babylonische Urkönig (Enmeduranki) steht an siebenter Stelle.

4. Ebenso bezeichnet der Name Jered (Irad) ein Fest: Das Hinabsteigen. Der babylonische Kalender kennt das Hinabsteigen (Urrad) des Gottes Nergal in die Unterwelt im vierten Monate, zur Zeit der Sommersonnenwende. Danach würde dieser Urvater beim Jahwisten an der richtigen Stelle stehen ${ }^{2}$. Das Hinabsteigen in die Unterwelt ist auch der griechischen Mythologie bekannt. Es ist möglich, daB sich dort die verlorene Legende von dem hinabgestiegenen Urvater Jered erhalten hat.

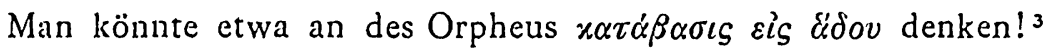

5. Vom fünften Urvater kennen wir nur den Namen oder vielmehr zwei Namen Mehujael und Mahalalel. Der eine würde an das Ende des Urvaters erinnern ${ }^{4}$. Der andere scheint wieder auf den Kultus (auf einen religiösen Lobgesang) hinzuweisen.

6. Handelt es sich danach bei Mahalalel vielleicht um irgendein - Freudenfest, so mag Methuselah der Legende eines Festes der Klage angehören. Auf die Gestalt dieses Urvaters fällt nämlich ein freilich unsicheres Licht von der phrygischen (lykaonischen) Urgeschichte her. In Ikonion lebte nach STEPHAN vON BYZANZ vor der Flut der Urvater Nannakos. Er hat das höchste Alter erreicht ${ }^{5}$. Ein Orakel verkündete, daß nach seinem Tode alle umkommen sollten. Deshalb

I Zimmern aaO. 330.

2 Döch muß nicht gerade an dieses Fest gedacht werden. Der Monat Ab wird als Monat des Herabsteigens des Feuergottes bezeichnet: Zimmern aaO. 417, Anm. 4. Auch andere Götter und Helden - insbesondere auch Ištar und ihr Befreier - steigen in die Unterwelt.

s Sie erinnert an die Höllenfahrt und dic Zurückführung der Ištar, ist aber bereits aus dem Gottlichen ins Menschliche ubertragen: Eurydike ist ein sterbliches Weib. Die Geschichte ihres Todes würde eine merkwurdige Lủcke in der biblischen Urgeschichte ausfullen: Eurydike ist auf eine Schlange. getreten und von ihr in die Ferse gebissen worden. Das sieht aus wie die Erfüllung des gütlichen Wortes in Gen 3 Is. Vgl. hiezu Gruppe, Griech. Myth., S. 875, Anm. 4. Auch die arkadische Urgeschichte vergißt der Schlange nicht. Doch ist es dort nicht ein Weib, sondern einer der Urkónige selbst, den eine Schlange tötet. Pausaxins VIII, 4, 4.

+ Oben bei Seite 75, Anm. 7.

s Über dreihundert Jahre. Wir durfen diese Zahl, die als eine besonders hohe gedacht ist, selbstverstandlich nicht mit den Altersangaben der Priesterschrift vergleichen. Sehr gut stimmt hier wieder die arkadische Urgeschichte zur lykaonischen, vgl. EpHorus bei Censorinus, De die natali c. I7 (Arcades dicere, reges aliquot ad trecentos vixisse annos), ebenso Plinius, Nat. Hist. 7, 49 mit der an die theologische Apologetik erinnernden Erklärung, die Jahre der Arkader seien dreimonatig (trimestres) gewesen. 
wehklagten die Phryger so, daß das Nannakos-Weinen ${ }^{\mathrm{I}}$ sprichwörtlich geworden ist. Wirklich ist gleich nach seinem Tode die deukalionische Flut hereingebrochen. Aucli Methušelah ist, wie die Rechnung ergibt, gerade im Jahre der Sintflut gestorben ${ }^{2}$.

7. Sicher ist die Gestalt Lamechs mit einem Feste, und zwar mit einem Erntefeste, bei dem Menschenopfer fielen, verknüpft. Das ist bereits in der ersten Abhandlung ${ }^{3}$ gezeigt worden.' Ebenso gewiß ist, daß dieser Urvater nicht an der'richtigen, dem Kalender entsprechenden Stelle steht.

8. Noah trägt, wie wir nun wissen, den Namen eines babylonischen Festes: es ist die Beruhigung (Nuhhu) des Herzens der erzürnten Götter. Die Erzählung von der Sintflut ist die Legende dieses Festes. Der Sintflutheld selbst ist in der Genesis und bei BERosus der zehnte Urkönig ${ }^{4}$. Das Fest wird also wohl einmal im zehnten Monate gefeiert worden, sein. Der Name dieses Monats (tebetu) mag die Arche bezeichnet haben ${ }^{5}$. Der Wassermann war im Stierzeitalter das zehnte Sternbild des Tierkreises ${ }^{6}$.

Die Urgeschichte ist also nicht eine Sammlung von volkstümlichen Sagen, Märchen oder Heldenliedern. Vielmehr hat dereinst priesterliche Gelehrsamkeit aus heiligen Texten, insbesondere aus Festlegenden, eine zúsammenhängende Urgeschichte der Menschheit, eine wahre Bibel vor der Bibel, geschaffen, bestimmt, uns über Opfer jeder Art, insbesondere auch über Menschenopfer, über Grund und Ritus der éinzelnen Feste, über die Ehe und nebenher auch über die ältesten profanen Erfindungen $\mathrm{zu}$ belehren. Das alttestamentliche Geschichtsbuch hat uns in einem knappen Auszuge jene ältere Vorläuferin, die Urbibel, aufbewahrt, obwohl sie einem andern Volke, einer andern Religion, einer andern Gesittung angehört. Und jene Urbibel ist trotz ihrer Barbarei (Menschenopfer, Blutschande) auch darin eine Vorläuferin der heiligen Schrift gewesen, daß sie von Land $z u$ Land gewandert ist und als eine wertvolle Geschichtsquelle, eine Urkunde über die Urgeschichte der

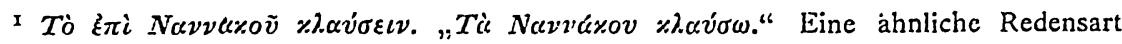
bei Sach $12_{\text {II }}$ : Die Klage um Hadad-Rimmon. Ramman ist der Herr der Sintflut: ZLMMERN aaO. 448.

2 Vgl. die Tabelle bei BuDDE, Bibl. Urgeschichte, S. 92. Daß ein Zeitalter (saeculum) mit dem Tode eines bestimmten Menschen schließt, entspricht ctruskischer Lehre. Censorinus aaO. ' Thain und Lamech, ZAW 35, ro.

4 Vgl. auch Jeremias aaO. Iot (indische Sage). ' 'Zimmern aaO. 547.

- Bedeutsam könnten auch sein dic Zwillinge als $z$ weites Sternbild (Kain und Abel) und der Schütze als achtes (Methušclah als .Mann des Wurfgeschosses ${ }^{u}$ ). Andere mannt liche Personen kommen, im Tierkreise nicht vor. 